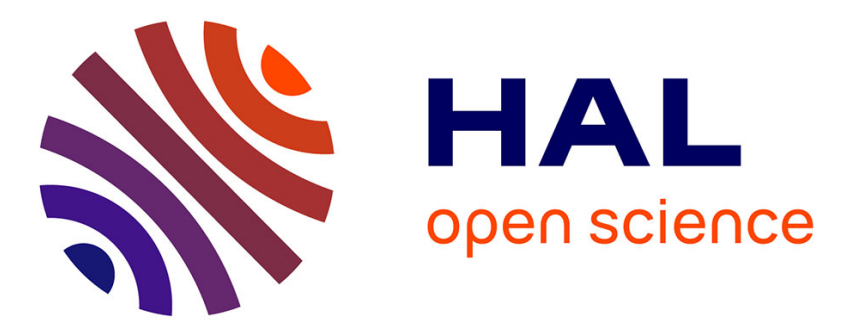

\title{
Line bisection in left neglect: The importance of starting right.
}

Marika Urbanski, Paolo Bartolomeo

\section{To cite this version:}

Marika Urbanski, Paolo Bartolomeo. Line bisection in left neglect: The importance of starting right.: The importance of starting right. Cortex, 2008, 44 (7), pp.782-93. 10.1016/j.cortex.2007.04.001 . inserm-00147591

\section{HAL Id: inserm-00147591 https://www.hal.inserm.fr/inserm-00147591}

Submitted on 23 May 2008

HAL is a multi-disciplinary open access archive for the deposit and dissemination of scientific research documents, whether they are published or not. The documents may come from teaching and research institutions in France or abroad, or from public or private research centers.
L'archive ouverte pluridisciplinaire HAL, est destinée au dépôt et à la diffusion de documents scientifiques de niveau recherche, publiés ou non, émanant des établissements d'enseignement et de recherche français ou étrangers, des laboratoires publics ou privés. 


\title{
Line bisection in left neglect:
}

\section{The importance of starting right}

\author{
Marika Urbanski ${ }^{1,2}$ and Paolo Bartolomeo ${ }^{1,3}$ \\ ${ }^{1}$ INSERM Unit 610, Hôpital de la Salpêtrière, Paris, France \\ ${ }^{2}$ Université de Grenoble, France \\ ${ }^{3}$ Department of Neurology, AP-HP, IFR 70, Hôpital de la Salpêtrière; Université
}

Pierre et Marie Curie-Paris6, Paris, France

Address correspondence to:

\author{
Paolo Bartolomeo \\ INSERM U 610 \\ Pavillon Claude Bernard \\ Hôpital Salpêtrière \\ 47 bd de l'Hôpital \\ F-75013 Paris - France \\ phone $+33(0) 142160025$ or 58 \\ FAX +33 (0) 142164195 \\ Email: paolo.bartolomeo@chups.inserm.fr \\ Web: http://marsicanus.free.fr/
}

Running head: The importance of starting right

Acknowledgments. We are grateful to the participants for their patience and good will.

Drs M. Blanchard, C. Jény and K. Moreau, and the staff of the Hôpital National de SaintMaurice, France, were of great help and assistance for patient referral and testing. We also thank C. Oppenheim, J-F. Meder, and the Department of Neuroimaging of the Ste-Anne Hospital, M. Thiebaut de Schotten, E. Volle, J. Chiras and the Department of Neuroimagery of the Salpêtrière Hospital for help with MRI scans. Results from this study were presented at the 23rd European Workshop on Cognitive Neuropsychology, Bressanone, Italy, January 2005. 


\section{ABSTRACT}

When marking the subjective midpoint of a horizontal line, patients with left unilateral neglect typically deviate rightward. Different accounts of this pattern of performance refer either to a biased competition between the two hemisegments of the line, with the right part being subjectively perceived as longer than the left part, or to a distortion of a cognitive representation of space, with spatial coordinates progressively relaxing from the right to the left. These accounts make different predictions about the role of the right part of the line, which is crucial in the biased competition account, but less important in the distortion account. To test these predictions, we asked participants to set the endpoints and the centre of perceived and imaginary lines. Contrary to previous studies, we controlled for the direction of performance of the endpoint task, with left-to-right trials and right-to-left trials being performed in separate blocks. Five patients with right hemisphere lesions and left neglect demonstrated the typical asymmetries when a right-sided stimulus (segment or endpoint) was present, but showed either normal performance or a reversed (leftward) bias while setting the endpoints and the centre of an imaginary line starting from the left side, when no right-sided visual stimulus was present until completion of each trial. We concluded that the right-sided portion of the line has a crucial importance in determining patients' rightwards deviations in line bisection, consistent with the biased competition hypothesis and with neurocognitive models of attentional orienting.

Keywords: Endpoint task, Spatial attention, Space representation, Brain damage 


\section{Introduction}

Patients with right hemisphere damage and left unilateral neglect typically bisect horizontal lines to the right of the true midpoint (Schenkenberg et al., 1980). A large body of evidence indicates that this pattern of performance can not be completely explained by elementary sensory deficits (Fuchs, 1920; D'Erme et al., 1987; Barton and Black, 1998), or by deficits in programming hand movements towards the left side (Milner et al., 1993), although such problems may well contribute to the patients' final performance (D'Erme et al., 1987; Bisiach et al., 1990; Doricchi and Angelelli, 1999; Doricchi et al., 2005). According to Marshall and Halligan (1990), during line bisection neglect patients search the line for its midpoint from the right to the left, and subsequently place the bisection point where the two hemi-segments appear to be of equal length. Eye-movement recordings confirmed that neglect patients adopt a right-to-left scanning strategy, and often fail to reach the leftmost extremity (Ishiai et al., 1992; Ishiai et al., 1989).

\section{1. Line bisection and orienting of spatial attention}

These notions are consistent with the possibility that rightward line bisection errors in neglect result from an overestimation of the right portion of the line as compared to the left portion. This perceptual asymmetry might be related to a bias in attentional orienting, favouring rightwards movements of attention, impairing leftward orienting, or both (see Bartolomeo and Chokron, 2002b, for review). Such an attentional bias might increase the perceptual salience of the right portion relative to the left portion of the line (Anderson, 1996; Bultitude and Aimola Davies, 2006). Thus, in line bisection the right and the left portion of the line would compete with each other until the point of subjective equality is reached. In left neglect, competition would be biased, causing the right portion to be overestimated with respect to the left portion. This, in turn, would bias the patients' response towards the right. The dysfunction of a right-hemisphere parietal-frontal network 
seems to be the lesional correlate of this biased competition (Thiebaut de Schotten et al., 2005; Doricchi and Tomaiuolo, 2003; Bartolomeo, 2006).

\section{2. The endpoint task}

A further important contribution to the understanding of line bisection performance in neglect came from the "endpoint task" devised by Bisiach and co-workers (Bisiach et al., 1994; Bisiach et al., 1996). After having performed a traditional bisection task, patients were presented with a single dot on a paper sheet and told that it was the centre of an imaginary line, similar to those they had been bisecting before. Patients had to mark the right and left endpoints of the imaginary line. Results showed that the distance between the left endpoint and the centre was greater than the distance between the centre and the right endpoint. Thus, patients tended to reproduce their line bisection errors (although the rightward deviation of the midpoint resulted smaller than in traditional line bisection ${ }^{1}$, an often disregarded detail). According to Bisiach and co-workers (1994), no existing hypothesis could account for this result, because neither right attentional capture, nor directional hypokinesia could explain the fact that patients travelled too far towards the left side when placing the left endpoint. Instead, Bisiach and co-workers (1996) interpreted their results as suggesting a distortion of spatial representation in left neglect. Spatial coordinates would display a progressive relaxation from the right to the left, such that patients should travel further leftward in order to equalize the amount of perceived spatial extent of the right and left (virtual) segments. According to this hypothesis, the mental representation of space would lose its symmetry with neglect, and become anisometric, because the distances between space coordinates would not be anymore uniform for the whole space, but would progressively increase from the right to the left. Alternatively,

\footnotetext{
${ }^{1}$ Neglect patients' mean rightward deviation was $9.33 \%$ for traditional bisection and $5.36 \%$ for the endpoint task, as calculated from Table 3 in Bisiach et al. (1996).
} 
however, patients' asymmetry of performance, with a right-sided (virtual) segment shorter than its left counterpart, also remains consistent with the previously described hypothesis of a perceptual asymmetry, leading to an overestimation of the right imagined segment in the context of a competition between the right and the left portions of the imagined line (Marshall and Halligan, 1990; Anderson, 1996).

\section{3. Levels of impairment: attentional vs. representational}

A crucial difference between the anisometry hypothesis and the competition hypothesis lies in the contribution of the right portion of the line to patients' final performance. According to the competition hypothesis, the right portion of the (virtual or physical) line has an obvious importance in shaping patients' performance, which is based on a perceptual comparison between the two subjective portions of the line. According to the anisometry account, on the other hand, the presence or absence of the right portion should not influence the overshoot of the left endpoint, which results from a relaxation of the relative spatial coordinates.

Consistent with the last prediction, Ishiai and his co-workers collected evidence suggesting that the right portion of the line is indeed unimportant to patients' performance. In a first study (Ishiai et al., 2000), neglect patients bisected lines by pointing on a touch panel with a pencil-like pointer. Lines of different length $(100,150$ or $200 \mathrm{~mm})$ were presented in a random order. There were three conditions: (1) traditional bisection; (2) bisection with cueing, in which patients had to bisect the line after having touched its left endpoint, in order to ensure their perception of the entire line length; (3) "representational" bisection, in which the line disappeared just after the patient had correctly touched its left endpoint; soon afterwards, patients had to touch the midpoint of the just vanished line. Seven patients out of eight deviated more on traditional bisection than on left-cued bisection, consistent with the well-known decrease of rightward errors after left cuing 
(Riddoch and Humphreys, 1983). Interestingly, the "representational" condition evoked similar or greater errors than the left-cueing condition, suggesting that after left cueing "the presence of the right segment... had no effect on bisection performance" (p. 749), and that "left neglect occurs mainly for the mental representation" of the line (p. 745). In a further study, Ishiai and co-workers (2004) asked patients to perform a similar "representational" task. Yet, after having touched the left endpoint, patients were now asked, in different trials, to touch either one of the endpoints, or the midpoint of the vanished line. Two patients out of three deviated more rightward on representational bisection than on cued bisection, confirming the previous results and the apparent irrelevance of the right segment of the lines to the final performance. However, it must be noted that several different line lengths were used; as a consequence, patients needed the visual information just presented in order to process each particular line after its disappearance. Thus, the only possible way for patients to perform these "representational" tasks was to make a bisection judgment on the physically presented line before it disappeared. After the line vanished, patients presumably placed the midpoint (or the endpoints) where they remembered them to have been on the physical line. If so, the task could hardly be considered as being purely “representational". Its outcome would rather reflect the patients' (biased) perception of the physical line, including, perhaps crucially, its right portion.

\section{4. Description of the present study}

These findings raise questions important to constrain functional and neural models of normal space cognition and of left neglect, and to understand the relationships between visual perception and visual mental imagery. First, is patients' performance on line bisection modulated by the presence/absence of right visual stimuli? Second, how do patients process imagined lines, when the presence/absence of either endpoint is controlled for? Is their performance equivalent to that with physically presented lines (Ishiai et al., 
$2000 ; 2004)$ or can they attain more symmetrical levels of performance in the absence of right-sided, attention-capturing physical stimuli?

To address these issues, we devised a new test of bisection of printed and imagined lines. We asked normal participants and left neglect patients to set the midpoint and the endpoints of 200-mm lines physically printed on a sheet. In different conditions, we invited participants to perform the test either starting from the left or from the right extremity of the line. In separate blocks, we presented participants with blank sheets and asked them to imagine lines similar to those that they had seen previously, and to mark the endpoints and the midpoint of the imagined lines, again starting either form the left or from the right extremity of each imagined line.

\section{5. Predictions}

According to the perceptual competition hypothesis reviewed above (Marshall and Halligan, 1990; Anderson, 1996), right, non-neglected physical stimuli are important to trigger left neglect. Therefore, the competition hypothesis predicts greater rightward displacements on the perceptual conditions than on the imagery conditions in neglect patients. Deviation should be modulated by the physical salience of the right stimuli, because salience presumably influences the capacity of these stimuli to capture patients' attention. A maximal deviation is thus expected in the perceptual condition with right-toleft endpoint marking, because the pencil mark on the right endpoint should increase the saliency of the right portion of the line. The imagery conditions should engender less rightward deviation in the right-to-left scanning condition, because in this condition there is only a single right-sided endpoint, a less salient stimulus than the line segment present in the perceptual conditions ${ }^{2}$. Finally, deviation should be minimized or even reversed in the

\footnotetext{
${ }^{2}$ An important component of stimulus intensity is luminance contrast. There is an obvious difference in luminance contrast between the linear region of space occupied by a solid line
} 
imagery condition with left-to-right scanning. In this condition, patients see no physical right-sided stimuli (other than the sheet margin and the casual distractors present during standard clinical examination in ambient light), when they are placing the midpoint. On the other hand, during midpoint placement there is a left-sided stimulus (the left endpoint) which might in principle attract attention, and thus possibly reverse their bias. Accounts based on representational impairments (Ishiai et al., 2000; Ishiai et al., 2004), such as space anisometry (Bisiach et al., 1996), predict on the contrary the absence of any modulation of patients' performance by physically present right-sided stimuli. Patients should show similar degrees of processing asymmetry, no matter whether the lines are printed or imagined, or whether a right-sided endpoint is present or not. Still different predictions result from directional motor deficits (Heilman and Valenstein, 1979; Butter et al., 1988). Patients should undershoot their movements to place the left endpoints, especially in the right-to-left conditions. However, leftward hypometria per se should not determine any differences in performance between perceptual and imagery conditions requiring hand movements in the same direction.

\section{Methods}

\section{1. Participants}

Five right-handed patients with right brain damage ( 3 women and 2 men; mean age 69.58 years; SD, 10.96), showing signs of moderate to severe left unilateral spatial neglect on a battery of paper and pencil tests (Bartolomeo and Chokron, 1999) and rightward deviation on two standard tests of line bisection (Azouvi et al., 2002; Bartolomeo and Chokron, 1999), and ten right-handed healthy participants without neurological impairment (5 women and 5 men; mean age, 69.13 years; SD, 5.85) consented to participate in the segment, black on white background, and the same region with only the single black endpoint of an invisible (virtual) line segment. 
experiment, which was carried out by following the guidelines of the ethical committee of the Hôtel-Dieu Hospital in Paris. Figure 1 shows patients' lesions, and table 1 their demographical and clinical data. Visual fields were assessed by Goldmann perimetry in all patients except MAL, who on clinical confrontation test often showed a "magnetic" gaze attraction towards right-sided stimuli (Gainotti et al., 1991). On those trials in which MAL was able to maintain fixation, however, she detected all left-sided stimuli, both on unilateral and on bilateral presentation, but sometimes reported to have seen a right-sided stimulus when presented with a left-sided one (allochiria).

Fig. 1 and Table 1 about here

\subsection{Stimuli}

For each trial of the perceptual conditions, a single horizontal black line (200-mm long, 1mm thick) was printed in the centre of a horizontal A4 sheet. A single line length was used to allow participants to memorize the line in order to perform the imagery conditions. For the imagery conditions, participants were presented with blank horizontal A4 sheets.

\subsection{Procedure}

There were four experimental conditions, preceded by a block of traditional line bisection. Two conditions involved physically presented lines (perceptual conditions): (1) traditional bisection, (2) left-to-right ( $\mathrm{L}>\mathrm{R}$ ) scanning and (3) right-to-left $(\mathrm{R}>\mathrm{L})$ scanning. The two remaining conditions employed imagined lines (imagery conditions): (4) left-to-right ( $\mathrm{L}>\mathrm{R})$ scanning and (5) right-to-left $(\mathrm{R}>\mathrm{L})$ scanning. Each condition consisted of 24 trials. Participants were presented with A4 paper sheets, in landscape format, centred on their trunk sagittal midline, at a viewing distance of approximately $40 \mathrm{~cm}$. They were requested to mark with a pencil held in their right hand the locations of the endpoints and the 
midpoint of a physically present line (perceptual conditions), or of an imaginary line (imagery conditions). To ensure that participants received enough practice with printed lines of the appropriate line length, so that they could perform the imagery conditions as accurately as possible, the experimental conditions were preceded by a block of traditional bisection of 200-mm lines $(n=24)$. In the following experimental blocks, each imagery condition was preceded by the corresponding perceptual condition, and conditions were blocked to maximize the correct application of the different task instructions. After the traditional bisection, JEC, MAL and MOB received first the $\mathrm{R}>\mathrm{L}$ conditions, then the $\mathrm{L}>\mathrm{R}$ conditions; the reverse order of experimental conditions was used for ANG and ROD.

\subsubsection{Perceptual conditions}

Participants performed first a traditional bisection task, in which they were requested to mark the location of the subjective midpoint of each presented line. In the perceptual $\mathrm{L}>\mathrm{R}$ condition, participants were presented with similar lines, but they were asked to mark with a pencil first the left endpoint, then the midpoint and finally the right endpoint of each line. The perceptual $\mathrm{R}>\mathrm{L}$ task followed the same procedure, with the opposite order of marking.

\subsubsection{Imagery conditions}

For each trial, participants were presented with a blank sheet and asked to conjure up the image of a line similar to those they saw in the previous condition. In different blocks, they were either invited to mark the endpoints and the midpoint in the $\mathrm{L}>\mathrm{R}$ order, or in the opposite $\mathrm{R}>\mathrm{L}$ order.

\subsection{Analysis of results}

Unilateral neglect is likely to be a heterogeneous condition, with different patients often demonstrating disparate patterns of performance. Therefore, we analyzed patients' performance both as a group, by comparing it with controls' performance, and individually, in a multiple single-case fashion. 


\subsubsection{Group study}

For each trial, the distance between each endpoint and the bisection point was measured to the nearest $\mathrm{mm}$. A deviation score was calculated for each trial by using the formula $\mathrm{L} /(\mathrm{L}+\mathrm{R})$, where $\mathrm{L}(\mathrm{R})$ indicates the mean distance from the subjective centre to the left (right) endpoint. Possible scores ranged from 0 (maximal leftward deviation, corresponding to the theoretical possibility of drawing the subjective centre on the left endpoint) to 1 (maximal rightward deviation). The scores were normalized by an arcsine transformation and entered in a repeated-measures analysis of variance (ANOVA), with group (controls, patients) as between-subjects factor and modality (perception, imagery) and scan direction $(\mathrm{L}>\mathrm{R}, \mathrm{R}>\mathrm{L})$ as within-subjects factors. This design implicated the exclusion of the traditional bisection condition, for which the scan direction factor could not be controlled. The $\mathrm{p}$ values were adjusted using the Greenhouse-Geisser correction, to compensate for possible violations of the sphericity assumption. For comparison purposes, we shall also give the more usual bisection measure corresponding to the deviation of the subjective centre relative to the true centre, in percentage of the mean line length for each condition, with negative signs for leftwards deviations and positive signs for rightwards deviations.

\subsubsection{Multiple single-case study}

We used the same measure as for the group study, i.e., the distance in mm between the subjective midpoint and each endpoint, and calculated the $95 \%$ inferential confidence intervals $(\mathrm{CI})$ for the mean of each condition. This graphical method addresses some of the problems of traditional null hypothesis testing (Tryon, 2001). For 200-mm lines, a 6.5-mm rightward shift of the subjective centre can be considered as a cutoff score between normal and pathological performance (Rousseaux et al., 2001; Azouvi et al., 2002). 


\section{Results}

Fig. 2 about here

\subsection{Group study}

Patients showed dramatic rightward deviations on the traditional bisection task (on average, $+41 \%$ of the line length) and on the $\mathrm{R}>\mathrm{L}$ perceptual condition ( $+30 \%)$ (Fig. 2$)$. The fact of setting the left endpoint before bisecting the line decreased patients' rightward shift to $+4 \%$, and placed it in the controls' range of performance for this condition. On the imagery conditions, patients still showed some rightward deviation $(+9 \%)$ when starting from the right endpoint; crucially, however, the fact of starting form the left endpoint rendered the performance of these patients with severe neglect similar to normal controls' (patients, $-1 \%$; controls, $+2 \%$ ). Consistent with these results, the ANOVA showed that all the principal effects and their interactions were reliable (group, $F(1,13)=9.96, P=0.007$; modality, $\mathrm{F}(1,13)=6.21, P=0.03$; scan direction, $\mathrm{F}(1,13)=18.69, P<0.001$; group $\mathrm{x}$ modality, $\mathrm{F}(1,13)=4.98, P=0.04$; modality $\mathrm{x}$ scan direction, $\mathrm{F}(1,13)=18.63, P<$ 0.001; group x modality x scan direction, $\mathrm{F}(1,13)=7.90, P=0.01)$. Post-hoc comparisons (Tukey HSD) showed that the perceptual R $>$ L condition performed by the patient group differed from all the remaining conditions (all $P s<0.008$ ). No other comparison reached significance ${ }^{3}$. Thus, patients performed differently from controls only when they had to scan a real line from the right extremity to the left, as predicted by the competition

\footnotetext{
${ }^{3}$ To assess a possible influence of the order of conditions, a further ANOVA was performed on the patients' data with block order $(\mathrm{L}>\mathrm{R}$ conditions first, $\mathrm{R}>\mathrm{L}$ conditions first) as additional factor. The block order did not reach significance, $\mathrm{F}(1,3)=1.28$, ns, nor did it interact with modality or scan direction, all Fs $<1$.
} 
hypothesis. Inspection of Fig. 2 indicates that patients' bias on the R $>$ L perceptual condition was similar to that shown on traditional line bisection, as revealed by the substantially overlapping CIs. This may suggest that patients often approach traditional bisection in a $\mathrm{R}>\mathrm{L}$ direction (Chokron et al., 1998). Fig. 2 also indicates that the imaginal $\mathrm{R}>\mathrm{L}$ condition gave rise to a lesser bias in the same direction. These results suggest that patients' performance was improved when either they scanned from the left or an imagined line was used instead of a real one. The scanning effect is most parsimoniously interpreted in terms of the well-established effects of leftwards attentional cueing. The imagery effect might reflect reduced right-sided competition for imagined vs. real lines; this may in turn account for the observation, originally made by Bisiach et al. (1996), that 'virtual lines' are bisected more accurately than real lines by neglect patients (see the present footnote 1).

\subsection{Multiple single-case study}

Participants' performance is displayed in figure 3. Non-overlapping CI indicate statistical difference $(\alpha=0.05)$ between the left and the right subjective segment.

Fig. 3 about here

Controls performed accurately and symmetrically in all conditions, with left-right asymmetries inferior to $3 \mathrm{~mm}$, with the exception of the $\mathrm{R}>\mathrm{L}$ imagery condition, where they drew right segments $5.59 \mathrm{~mm}$ longer than their left counterpart. However, CI overlap (Fig. 3) indicates that the difference, corresponding to a $-3 \%$ deviation of the subjective centre, is not statistically reliable.

All the patients showed right-directed deviations especially or exclusively when the scanning direction was from the right to the left. In traditional bisection, mean rightward 
deviations ranged from $14 \%$ to $70 \%$. Results of the remaining experimental conditions are described below.

\subsection{Perception, $R>L$}

Performance was similar to traditional bisection, with rightward shift ranging from $13 \%$ to $65 \%$. JEC and, to a lesser extent, MAL often failed to reach the true left endpoint of the line. This undershooting may reflect either a directional motor disorder, with leftward hypometria (Heilman and Valenstein, 1979), or a failure to compensate for the visual field defect (Doricchi and Angelelli, 1999; Doricchi et al., 2005). The last possibility seems more probable, given that in the perceptual $\mathrm{L}>\mathrm{R}$ condition JEC was able to reach the left extremity of the lines (on the other hand, on this condition she sometimes did not reach the right endpoint). Although MAL could detect left-sided stimuli on visual confrontation test, her magnetic gaze attraction toward the rightmost visual stimulus may have prevented proper processing of the left extremity of the printed lines. Leftward undershooting is obviously inconsistent with a simple anisometry account, which was originally intended to explain leftward overshooting.

\subsubsection{Perception, $L>R$}

All the patients deviated rightward, albeit to a lesser extent than in the $\mathrm{R}>\mathrm{L}$ condition (range, $+4 \%$ to $+22 \%$ ), except MAL, who deviated leftwards by $21 \%$. Thus, in this patient starting from the left endpoint actually reversed the performance bias. Leftward (reversed) bisection errors are described in left neglect patients after cueing of the left endpoint (Mattingley et al., 1993). A dependency on the starting endpoint has been described in two neglect patients who performed a passive bisection task by stopping a dot travelling on a line (either leftward or rightward), upon its crossing the centre (Chokron et al., 1998), and is consistent with the proposal that neglect patients' errors depend on the direction of their scanning of the line (Marshall and Halligan, 1989). A compensatory left-sided scanning 
strategy, in association with a non-lateralised attentional loss, may lead to right inattention in left neglect patients on tasks of line bisection, drawing and multiple task cancellation (Robertson et al., 1994).

\subsubsection{Imagery, $R>L$}

Again, all the patients placed the midpoint rightward of the true midpoint, but deviated less (range, $+3 \%$ to $+15 \%$ ) than in the corresponding perceptual condition, consistent with the idea that the right-sided line segment present in the perceptual condition can capture patients' attention more effectively than the (less salient) right endpoint used in this condition.

\subsubsection{Imagery, $L>R$}

Crucially, in this condition there was no more rightward shift in patients' performance. Instead, different individual patterns emerged. Patients ROD, MOB and ANG performed quite symmetrically (respectively, $+1 \%,+2 \%$ and $+4 \%$ ), well in the range of controls' performance, which was between $-3 \%$ and $+5 \%$ for this condition. There is a striking contrast between these patients' rightwards-biased performance when right-sided visual stimuli were present, and their normal performance when no right-sided stimulus was visible until completion of each trial. Also patients JEC and MAL did not demonstrate any rightward deviation on this condition; they showed instead a leftward shift (respectively $7 \%$ and $-4 \%$ ), again consistent with the proposed dependency of patients' performance on their scanning direction (Marshall and Halligan, 1989; Chokron et al., 1998).

\section{Discussion}

\subsection{Summary of results}

In this study, we aimed to clarify whether rightwards deviations on line bisection in left neglect patients depended or not on the physical presence of the right portion of the line. We asked participants to bisect perceived lines, or to set the endpoints and the centre of 
imagined lines. Contrary to previous studies, we controlled for the direction of performance of the endpoint task, with left-to-right trials and right-to-left trials being performed in separate blocks. Five consecutive patients with focal lesions in the right hemisphere, included on the basis of their having left neglect and rightwards deviations on line bisection, had either normal performance or a reversed (leftward) bias when setting the endpoints and the centre of an imaginary line starting from the left side. In this condition, no visual stimulus was present on the right side until each trial was completed. Furthermore, the salience of these stimuli predicted to some extent the magnitude of deviation, with more deviation for more salient stimuli, such as a line segment, and less deviation for less salient stimuli, such as a mere endpoint marker. This is consistent with the results of the seminal study with the endpoint task (Bisiach et al., 1996), where there was also more deviation with solid lines than with endpoints only (see the present footnote 1).

\subsection{Test of predictions}

These results are in line with the predictions stemming from models based on a biased competition between right- and left-sided perceptual stimuli. Alternative explanations, implicating either "representational" deficits (Ishiai et al., 2000; Bisiach et al., 1996) or directional motor deficits (Heilman and Valenstein, 1979; Butter et al., 1988), fail to account for the observed dissociation in performance between tasks with or without rightsided visual stimuli. Of course, our data do not eliminate the possibility that these deficits play a role in other patients' patterns of impairment. All they suggest is that representational or pre-motor deficits cannot be invoked as a general mechanism of line bisection performance. We were impressed by finding that the mere fact of starting the task from the left endpoint was able to nullify or even reverse neglect-related behaviour in patients with severe neglect resulting from extensive right hemisphere damage. The 
observed pattern of results is consistent with the recent observation that line bisection behaviour is especially influenced by the location of the right endpoint of the line (McIntosh et al., 2005).

When placing the left endpoint in the right-to-left imagery condition, patients ROD, MOB and ANG travelled too far towards the left, producing virtual left-sided segments longer than $100 \mathrm{~mm}$ (see Fig. 3), similar to the overshooting behaviour described by Bisiach and associates in their seminal endpoint study (Bisiach et al., 1996). This pattern of performance was subsequently found typical of patients showing an association of neglect and hemianopia (Doricchi and Angelelli, 1999). In support of this hypothesis, ROD, MOB and ANG were the only three patients in our series showing left hemianopia. As noted by Bisiach et al. (1996) this behaviour is opposite to that predicted by accounts based on directional motor deficits, such as directional hypokinesia or hypometria (Heilman and Valenstein, 1979; Butter et al., 1988). Importantly, however, ROD, MOB and ANG demonstrated no asymmetry of performance in the $\mathrm{L}>\mathrm{R}$ imagery condition. This result runs counter the spatial anisometry account (Bisiach et al., 1996), because distortions of spatial coordinates should not depend on the presence or absence of a right-sided visual stimulus. Instead, leftward hypermetria may be understood as a consequence of saccadic overshooting made to move the blind hemifield away and to bring the endpoint position into the seeing hemifield (Doricchi and Angelelli, 1999; Doricchi et al., 2003). This process might result in patients "confabulating" a longer left segment (Chatterjee, 2002).

Traditionally, perceiving an object is conceived as building a mental representation of the object. Spatial attention can thus have consequences on representation, in the sense that an unattended object will not be represented in the same form than an attended object. If so, then accounts of patients' performance in terms of attentional bias, representational deficit or directional hypokinesia would not be mutually exclusive, and the present results 
would simply speak to the issue of how horizontal linear representations are modifiable in neglect by different factors, such as directional scanning, perception or memory. In our opinion, the reader should be free to interpret the results in this way. We note, however, that this view is attracting increasing criticism. For example, theorists of active or animate perception maintain that "seeing is a way of acting. It is a particular way of exploring the environment. Activity in internal representations does not generate the experience of seeing. The outside world serves as its own, external, representation" (O'Regan and Noë, 2001, p. 939; see also Ballard et al., 1997). In this perspective, we argue that in the neglect patients we tested, it is the interaction with the outside world that is impaired, not putative "internal representations". Together with many other similar results reviewed below, the present data warn against hasty interpretations of neglect patients' patterns of performance as "representational distortions".

\subsection{Relation to previous "imaginal" bisection results}

The remarkable work of the Ishiai group on eye movements in line bisection (Ishiai et al., $1989 ; 1996 ; 2006)$ greatly helped to shape our present conclusions. The studies of Ishiai et al. $(2000 ; 2004)$ were a direct source of inspiration for the present experiments. Although the present demonstration of the importance of right-sided visual stimuli in bisectionrelated tasks suggests that the "representational" conditions in these studies may have been contaminated by visual perception, our results fully support Ishiai's conclusion that, in line bisection, "[t]he left extent [of the line] seems to be inferred mainly from the attended right extent" (Ishiai, 2002, p. 191).

Using the endpoint task, Nico et al. (1999) had patients mark either the left or the right endpoint of a virtual line of which the centre and the other endpoint were given. A group of ten neglect patients generally underestimated the distances. Collapsing the results of the different trials for each given endpoint, left endpoints were marked farther from the 
centre than right endpoints ${ }^{4}$. In the light of our findings, it might be that this asymmetry of performance essentially resulted from patients travelling farther away from the starting point when the right endpoint and the centre were given, as compared to the opposite, $\mathrm{L}>\mathrm{R}$ condition. A general, non-lateralized tendency to produce shorter lines (perhaps reflecting motor hypometria or perceptual overestimation) may have contributed to the final pattern of results.

In a study devoted to crossover (the tendency of left neglect patients to make leftdirected errors when bisecting shorter lines), Mennemeier et al. (2005) presented a line for 10s, then withdraw the line, gave participants a blank sheet with a single endpoint, and asked them to set the relative midpoint from memory. When starting from the right endpoint, neglect patients deviated rightwards with long imagined lines and leftwards with short imagined lines, consistent with their performance with physical lines. Starting from the left endpoint, however, reversed the sense of patients' errors. These results are similar to the performance of patients JEC and MAL, who showed a reversed (leftward) bias with imagined lines in the $\mathrm{L}>\mathrm{R}$ condition, and are not inconsistent with the performance of the three remaining patients of the present study, whose rightward bias was nullified by the fact of starting from the left endpoint. Our results generalize those of Mennemeier et al. to the case where the patients marked not only the midpoint of the virtual line, but also its endpoints, and address more directly the issue of mental imagery, because our participants did not see a physical line before each imaginal bisection; consequently, they could not make a bisection judgment on the physically presented line before it disappeared. Overall,

\footnotetext{
${ }^{4}$ At variance with the Bisiach et al's (1996) results, but similar to the performance of our patients JEC and MAL, the subjective distance from the centre was always shorter than the real distance.
} 
our results confirm Mennemeier et al.'s conclusion of an important role of a rightward attentional bias in shaping neglect patients' performance in line bisection.

\subsection{Relation to attentional impairments in neglect}

The present results support the hypothesis that line bisection performance results from the competition between the subjective lengths of the two line segments (Marshall and Halligan, 1990; Anderson, 1996). In left neglect patients, whose attention is right-biased, the right segment may subjectively appear as longer than the left segment (see Milner and Harvey, 1995). As a consequence, the left extremity of the right segment, which corresponds to the centre of the line, may be displaced rightward, thus giving rise to the bisection error. Left hemianopia may further decrease the saliency of the left segment, thus aggravating the rightward bias (Bartolomeo, 1987; D'Erme et al., 1987; Doricchi et al., 2005). Conversely, a reduction or absence of right-sided stimuli, as in the present imagery conditions, may reduce or abolish the rightward bias. The importance of starting from the right endpoint in neglect patients' line bisection performance is also underlined by eye movements studies, showing that patients' fixations often land on the right part of the line (Ishiai et al., 1989). This preferential scanning direction is nicely illustrated by the funny drawings which a distinguished artist with neglect could not help adding to his bisections (Cantagallo and Della Sala, 1998) (Fig. 4a).

Fig. 4 about here

Even when patients manage to look at the left extremity of the line, the final bisection point remains rightward-shifted (Ishiai et al., 1996; 2006). In these cases, and despite the (ineffective) leftward saccades, the weight of attention appears to rest on the right portion 
of the line (Fig. 4b), as if this right-sided portion continued to exert a "magnetic" attraction on patients' attention (see Gainotti et al., 1991).

Rightward attentional capture shapes many aspects of neglect behaviour. In cancellation tasks, the number of left-sided omissions can be decreased by having patients erasing the stimuli, instead of cancelling them with a pencil mark (Mark et al., 1988), because the erased right-sided targets cannot attract patients' attention any more. In drawing common objects from memory, blindfolding decreases left omissions (Chokron et al., 2004), presumably because it nullifies the attention-capturing power of the right-sided details just drawn by the patient. Also the lesser frequency and severity of neglect in imaginal, as opposed to perceptual, tasks (Halsband et al., 1985; Bartolomeo et al., 1994; Manoach et al., 1996; Bartolomeo et al., 2005) suggests that right-sided imagined details have less "magnetic" power than their physical counterparts in visuo-spatial tests (Bartolomeo et al., 1994; Bartolomeo and Chokron, 2002a; Chokron et al., 2004). More relevant to line bisection, Chokron and her co-workers (1998) asked normal participants and neglect patients to stop a dot travelling on a line when it crossed its centre. The dot moved either from the left to the right or in the opposite direction. Results showed that the subjective midpoint depended on the direction of scanning, being shifted towards the starting point of the dot, as if participants' attention were attracted by the moving dot. Finally, when neglect patients had to mark on the horizontal margin of a sheet the approximate location indicated by a vertical arrow situated on the opposite margin (a task first proposed by Halligan and Marshall, 1991), they deviated rightward, but only when the possible response locations were indicated by physically presented stimulus (a line or a number); when patients had to mark a blank location on an empty margin, no deviation occurred (Bartolomeo et al., 2004). In this task, when visual stimuli were present patients' attention was presumably captured by the distractors rightward of the target, which biased 
their response in this direction; this could not be the case in the condition without visual stimuli, because nothing could capture patients' attention on the right side of the sheet.

Our evidence is also consistent with the typical performance of left neglect patients on cued response time tasks, where their responses to left targets are disproportionally slowed by the previous presentation of a right-sided, attention-capturing cue (Posner et al., 1984; D'Erme et al., 1992; Bartolomeo et al., 2001). Thus, the present account has the advantage of reconciling many sources of evidence showing that right-sided, nonneglected visual stimuli are important to trigger left neglect with the large body of research on line bisection, including the apparently conflicting findings reported by Bisiach and coworkers $(1994 ; 1996)$ and by Ishiai and co-workers'(2000; 2004).

\subsection{Relation to models of spatial attention}

Finally, the present findings are in line with neurocognitive models of spatial attention. Neuroimaging studies (Corbetta and Shulman, 2002) have demonstrated a right-lateralized, ventral parieto-frontal system important for stimulus-driven, or exogenous attention, which is the form of spatial attention typically impaired in unilateral neglect (Bartolomeo and Chokron, 2002b), whereas a more dorsal parieto-frontal system subserves goal-directed, or endogenous attention. Corbetta and co-workers (2005) recently demonstrated that in left neglect patients the dorsal system can show hemodynamic signs of relative overactivity of the left superior parietal lobule in comparison to its right counterpart. This imbalance, which is reminiscent of Kinsbourne's “opponent processors” model (Kinsbourne, 1993), can account for the tendency to rightward shifts of attention shown by neglect patients (see Gainotti et al., 1991). In the monkey, parietal areas such as LIP (Gottlieb et al., 1998) or 7a (Mesulam, 1999) are important for processing the salience of visual objects. Using intraoperative stimulation in neurosurgical patients, we recently demonstrated that rightward deviations on line bisection occurred upon temporary inactivation of the supra-marginal 
gyrus of the inferior parietal lobule and of the caudal portion of the superior temporal gyrus, but especially during the inactivation of a long-range pathway linking parietal and frontal regions (Thiebaut de Schotten et al., 2005). A parieto-frontal disconnection (Mesulam, 1999; Doricchi and Tomaiuolo, 2003; Gaffan and Hornak, 1997) may thus disrupt the symmetrical processing of the visual scene by biasing the competition for attention in favour of ipsilesional items (Bartolomeo, 2006), and produce the rightwardsbiased behaviour shown by neglect patients when right-sided visual objects are present in the visual scene.

Despite these considerations, we acknowledge that the available models of spatial attention remain insufficiently articulated to fully account for the present results. The increasingly better definition of anatomical and diffusion MRI, with the possibility to visualize disturbances of brain connectivity (Catani, 2006) in addition to cortical damage, coupled with detailed behavioural evidence from brain-damaged patients, may offer promising ways to bridge the gap between cognitive models and their neural implementation. 


\section{References}

ANDERSON B. A mathematical model of line bisection behaviour in neglect. Brain, 119: 841-850, 1996.

Azouvi P, Samuel C, Louis-Dreyfus A, Bernati T, Bartolomeo P, Beis J-M, Chokron S, Leclercq M, Marchal F, Martin Y, De Montety G, Olivier S, Perennou D, Pradat-Diehl P, Prairial C, Rode G, Sieroff E, Wiart L and ROUSSEAUX M. Sensitivity of clinical and behavioural tests of spatial neglect after right hemisphere stroke. Journal of Neurology, Neurosurgery and Psychiatry, 73: 160-166, 2002.

BALLARD DH, HAYHOE MM, POOK PK and RAO RPN. Deictic codes for the embodiment of cognition. Behavioral and Brain Sciences, 20: 723-767, 1997.

BARTOLOMEO P. Aspetti dell'emi-inattenzione spaziale nelle lesioni emisferiche: fattori che influenzano la bisezione di linee [Aspects of spatial hemi-inattention: factors influencing line bisection performance]. Unpublished MD Thesis, Rome: Catholic University, 1987.

BARTOLOMEO P. A parieto-frontal network for spatial awareness in the right hemisphere of the human brain. Archives of Neurology, 63: 1238-1241, 2006.

Bartolomeo P, BACHoud-LÉvi A-C, Azouvi P and Chokron S. Time to imagine space: a chronometric exploration of representational neglect. Neuropsychologia, 43: 1249-1257, 2005.

BARTOLOMEO P and CHOKRON S. Egocentric frame of reference: Its role in spatial bias after right hemisphere lesions. Neuropsychologia, 37: 881-894, 1999.

BARTOLOMEO P and CHOKRON S. Can we change our vantage point to explore imaginal neglect? (Commentary on Pylyshyn: Mental imagery: In search of a theory). Behavioral and Brain Sciences, 25: 184-185, 2002a.

BARTOLOMEO P and CHOKRON S. Orienting of attention in left unilateral neglect. Neuroscience and Biobehavioral Reviews, 26: 217-234, $2002 \mathrm{~b}$.

BARTOLOMEO P, D'ERME P and GAINOTTI G. The relationship between visuospatial and representational neglect. Neurology, 44: 1710-1714, 1994.

Bartolomeo P, Siéroff E, Decaix C and Chokron S. Modulating the attentional bias in unilateral neglect: The effects of the strategic set. Experimental Brain Research, 137: 424-431, 2001.

Bartolomeo P, Urbanski M, Chokron S, Chainay H, Moroni C, Siéroff E, Belin C and HALLIGAN P. Neglected attention in apparent spatial compression. Neuropsychologia, 42: 4961, 2004.

BARTON JJ and BLACK SE. Line bisection in hemianopia. Journal of Neurology, Neurosurgery and Psychiatry, 64: 660-2, 1998.

Bisiach E, Geminiani G, Berti A and Rusconi ML. Perceptual and premotor factors of unilateral neglect. Neurology, 40: 1278-1281, 1990.

Bisiach E, Pizzamiglio L, Nico D and Antonucci G. Beyond unilateral neglect. Brain, 119: 851-857, 1996.

Bisiach E, Rusconi ML, Peretti VA and Vallar G. Challenging current accounts of unilateral neglect. Neuropsychologia, 32: 1431-1434, 1994.

Bultitude JH and Aimola DAVIES AM. Putting attention on the line: investigating the activationorientation hypothesis of pseudoneglect. Neuropsychologia, 44: 1849-1858, 2006.

BUtTER CM, MARK VW and HEILMAN KM. An experimental analysis of factors underlying neglect in line bisection. Journal of Neurology, Neurosurgery and Psychiatry, 51: 1581-1583, 1988.

CANTAgallo A and Della Sala S. Preserved insight in an artist with extrapersonal spatial neglect. Cortex, 34: 163-189, 1998.

CATANI M. Diffusion tensor magnetic resonance imaging tractography in cognitive disorders. Current Opinion in Neurology, 19: 599-606, 2006. 
ChAtTerJee A. Spatial anisometry and representational release in neglect. In Karnath H.O., Milner D. and Vallar G. (Eds.), The Cognitive and Neural Bases of Spatial Neglect. Oxford: Oxford University Press, 2002, pp. 167-180.

Chokron S, Bartolomeo P, Perenin MT, Helft G and Imbert M. Scanning direction and line bisection: A study of normal subjects and unilateral neglect patients with opposite reading habits. Cognitive Brain Research, 7: 173-178, 1998.

CHOKRON S, COLLIOT P and BARTOLOMEO P. The role of vision on spatial representations. Cortex, 40 : 281-290, 2004.

Corbetta M, KinCAde MJ, Lewis C, SNyder AZ and SAPIR A. Neural basis and recovery of spatial attention deficits in spatial neglect. Nature Neuroscience, 8: 1603-1610, 2005.

CORBETTA M and SHULMAN GL. Control of goal-directed and stimulus-driven attention in the brain. Nature Reviews Neuroscience, 3: 201-215, 2002.

D'ERme P, DE Bonis C and GAINOTTI G. Influenza dell'emi-inattenzione e dell'emianopsia sui compiti di bisezione di linee nei pazienti cerebrolesi [Influence of unilateral neglect and hemianopia on line bisection performance in brain-damaged patients]. Archivio di Psicologia, Neurologia e Psichiatria, 48: 165-189, 1987.

D'ERme P, RoBertson IH, BARTOLOMEO P, DANiEle A and GAINOTTI G. Early rightwards orienting of attention on simple reaction time performance in patients with left-sided neglect. Neuropsychologia, 30: 989-1000, 1992.

DORICCHI F and ANGELELLI P. Misrepresentation of horizontal space in left unilateral neglect: role of hemianopia. Neurology, 52: 1845-1852, 1999.

DORICCHI F, GUARIGLIA P, Figliozzi F, MAGNOTTI L and GABRIELE G. Retinotopic modulation of space misrepresentation in unilateral neglect: evidence from quadrantanopia. Journal of Neurology, Neurosurgery and Psychiatry, 74: 116-119, 2003.

Doricchi F, GUARIGLIA P, Figliozzi F, SilvetTI M, BRUNO G and GASPARINI M. Causes of cross-over in unilateral neglect: between-group comparisons, within-patient dissociations and eye movements. Brain, 128: 1386-1406, 2005.

DORICCHI F and TOMAIUOLO F. The anatomy of neglect without hemianopia: a key role for parietalfrontal disconnection? NeuroReport, 14: 2239-2243, 2003.

FuCHS W. Untersuchung über das Sehen der Hemianopiker und Hemiamblyopiker. Zeitschrift für Psychologie und Physiologie der Sinnersorganen, 84: 67-169, 1920.

GAFFAN D and HORNAK J. Visual neglect in the monkey. Representation and disconnection. Brain, 120: 1647-1657, 1997.

GAinOtTI G, D'ERME P and BARTOLOMEO P. Early orientation of attention toward the half space ipsilateral to the lesion in patients with unilateral brain damage. Journal of Neurology, Neurosurgery and Psychiatry, 54: 1082-1089, 1991.

GotTLIEB JP, KUSUNOKI M and GOLDBERG ME. The representation of visual salience in monkey parietal cortex. Nature, 391: 481-484, 1998.

HALLIGAN PW and MARSHALL JC. Spatial compression in visual neglect: A case study. Cortex, 27: 623-629, 1991.

HALSBAND U, GRUHN S and ETTLINGER G. Unilateral spatial neglect and defective performance in one half of space. International Journal of Neurosciences, 28: 173-95, 1985.

Heilman KM and Valenstein E. Mechanisms underlying hemispatial neglect. Annals of Neurology, 5: 166-170, 1979.

ISHIAI S. Perceptual and motor interaction in unilateral spatial neglect. In Karnath H.O., Milner D. and Vallar G. (Eds.), The Cognitive and Neural Bases of Spatial Neglect. Oxford: Oxford University Press, 2002, pp. 181-193.

ISHIAI S, FURUKAWA T and TSUKAGOSHI H. Visuospatial processes of line bisection and the mechanisms underlying unilateral spatial neglect. Brain, 112: 1485-502, 1989. 
ISHIAI S, KOYAMA Y, NAKANO N, SEKI K, NiSHIDA Y and HAYASHI K. Image of a line is not shrunk but neglected. Absence of crossover in unilateral spatial neglect. Neuropsychologia, 42: 251-216, 2004.

Ishiai S, KoYama Y, SeKi K, HaYASHI K and IzUmi Y. Approaches to subjective midpoint of horizontal lines in unilateral spatial neglect. Cortex, 42: 685-691, 2006.

ISHIAI S, KOYAMA Y, SEKI K and IZAWA M. Line versus representational bisections in unilateral spatial neglect. Journal of Neurology, Neurosurgery and Psychiatry, 69: 745-750, 2000.

ISHIAI S, SEKI K, KOYAMA Y and GONO S. Ineffective leftward search in line bisection and mechanisms of left unilateral spatial neglect. Journal of Neurology, 243: 381-387, 1996.

Ishiai S, Sugishita M, Mitani K and IshiZAWA M. Leftward search in left unilateral spatial neglect. Journal of Neurology, Neurosurgery \& Psychiatry, 55: 40-44, 1992.

KINSBOURNE M. Orientational bias model of unilateral neglect: Evidence from attentional gradients within hemispace. In Robertson I.H. and Marshall J.C. (Eds.), Unilateral Neglect: Clinical and Experimental Studies. Hove (UK): Lawrence Erlbaum Associates, 1993, pp. 63-86.

MANOACH DS, O'CONNOR M and WEINTRAub S. Absence of neglect for mental representations during the intracarotid amobarbital procedure. Archives of Neurology, 53: 333-336, 1996.

Mark VW, KoOISTRA CA and HeILMAN KM. Hemispatial neglect affected by non-neglected stimuli. Neurology, 38: 640-643, 1988.

MARSHALl JC and HALLIGAN PW. When right goes left: An investigation of line bisection in a case of visual neglect. Cortex, 25: 503-515, 1989.

MARSHALl JC and HALLIGAN PW. Line bisection in a case of visual neglect: Psychophysical studies with implications of theory. Cognitive Neuropsychology, 7: 107-130, 1990.

Mattingley JB, Pierson JM, Bradshaw JL, Phillips JG and Bradshaw JA. To see or not to see: the effects of visible and invisible cues on line bisection judgements in unilateral neglect. Neuropsychologia, 31: 1201-1215, 1993.

MCINTOSH RD, SCHINDLER I, BIRCHALl D and MiLnER AD. Weights and measures: A new look at bisection behaviour in neglect. Cognitive Brain Research, 25: 833-850, 2005.

Mennemeier M, Pierce CA, Chatterjee A, Anderson B, Jewell G, Dowler R, Woods AJ, Glenn $\mathrm{T}$ and MARK VW. Biases in attentional orientation and magnitude estimation explain crossover: neglect is a disorder of both. Journal of Cognitive Neuroscience, 17: 1194-1211, 2005.

MESUlAm MM. Spatial attention and neglect: parietal, frontal and cingulate contributions to the mental representation and attentional targeting of salient extrapersonal events. Philosophical Transactions of the Royal Society of London B, 354: 1325-1346, 1999.

MiLner AD and HARVEY M. Distortion of size perception in visuospatial neglect. Current Biology, 5: 85-89, 1995.

Milner AD, HARVEY M, RoBERTS RC and ForSTER SV. Line bisection errors in visual neglect: Misguided action or size distortion? Neuropsychologia, 31: 39-49, 1993.

Nico D, GALATI G and InCOCCIA C. The endpoints' task: an analysis of length reproduction in unilateral neglect. Neuropsychologia, 37: 1181-1188, 1999.

O'REGAN JK and NOË A. A sensorimotor account of vision and visual consciousness. Behavioral and Brain Sciences, 24: 939-1011, 2001.

POSNER MI, WALKER JA, FRIEDRICH FJ and RAFAL RD. Effects of parietal injury on covert orienting of attention. Journal of Neuroscience, 4: 1863-1874, 1984.

RIDDOCH MJ and HUMPHREYS GW. The effect of cueing on unilateral neglect. Neuropsychologia, 21: 589-599, 1983.

Robertson IH, Halligan PW, Bergego C, Homberg V, Pizzamiglio L, Weber E and Wilson BA. Right neglect following right hemisphere damage? Cortex, 30: 199-213, 1994.

Rousseaux M, Beis JM, Pradat-Diehl P, Martin Y, Bartolomeo P, Chokron S, Leclercq M, Louis-Dreyfus A, Marchal F, Pérénnou D, Prairial C, SAmuel C, Siéroff E, Wiart L and AzOuvi P. Normalisation d'une batterie de dépistage de la négligence spatiale. Etude de 
l'effet de l'âge, du niveau d'éducation, du sexe, de la main et de la latéralité [Presenting a battery for assessing spatial neglect. Norms and effects of age, educational level, sex, hand and laterality]. Revue Neurologique, 157: 1385-1401, 2001.

SCHENKENBERG T, BRADFORD DC and AJAX ET. Line bisection and unilateral visual neglect in patients with neurologic impairment. Neurology, 30: 509-517, 1980.

Thiebaut de Schotten M, Urbanski M, Duffau H, Volle E, LÉvy R, Dubois B and Bartolomeo P. Direct evidence for a parietal-frontal pathway subserving spatial awareness in humans. Science, 309: 2226-2228, 2005.

TRYON WW. Evaluating statistical difference, equivalence, and indeterminacy using inferential confidence intervals: an integrated alternative method of conducting null hypothesis statistical tests. Psychological Methods, 6: 371-386, 2001. 


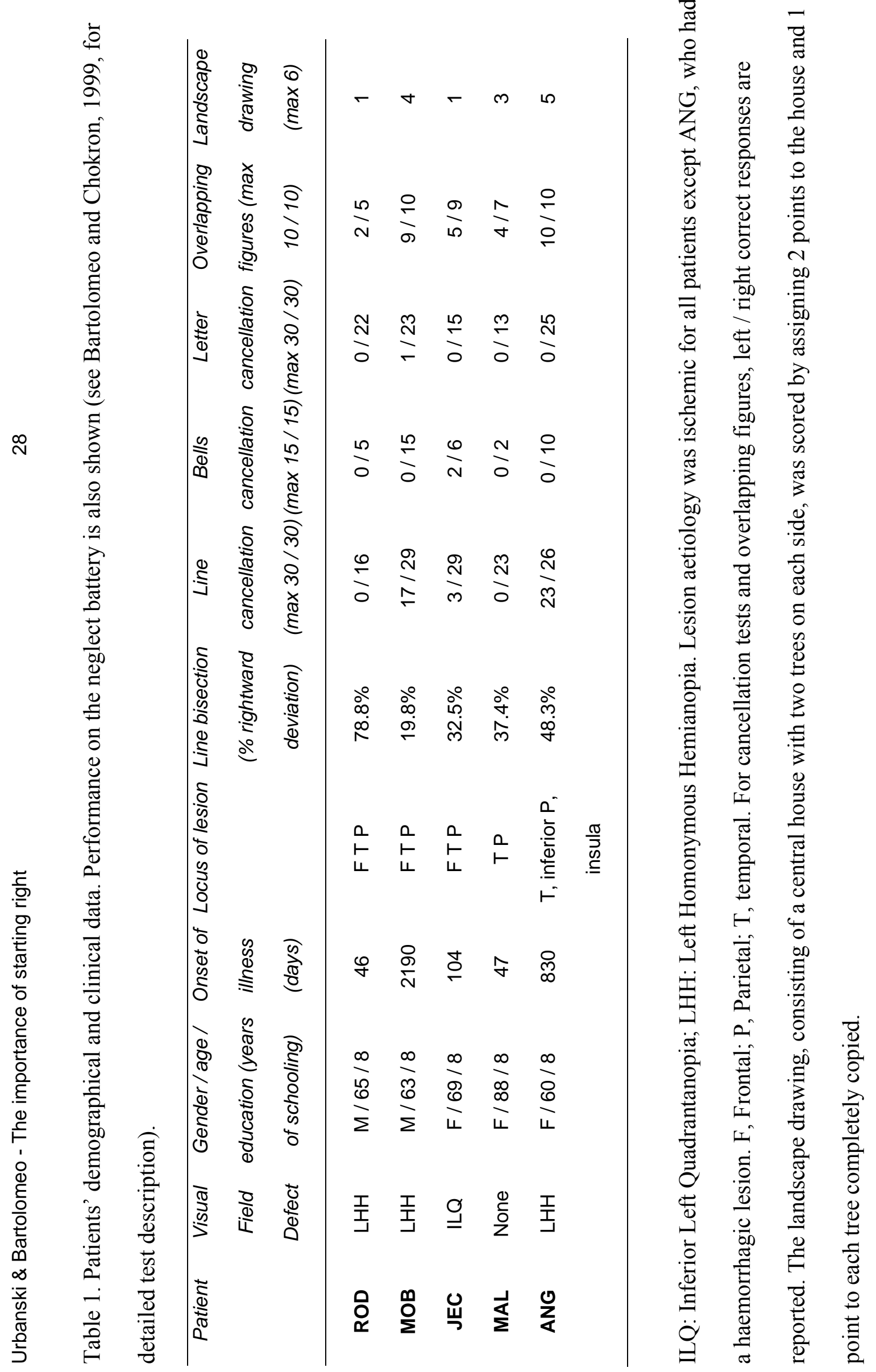




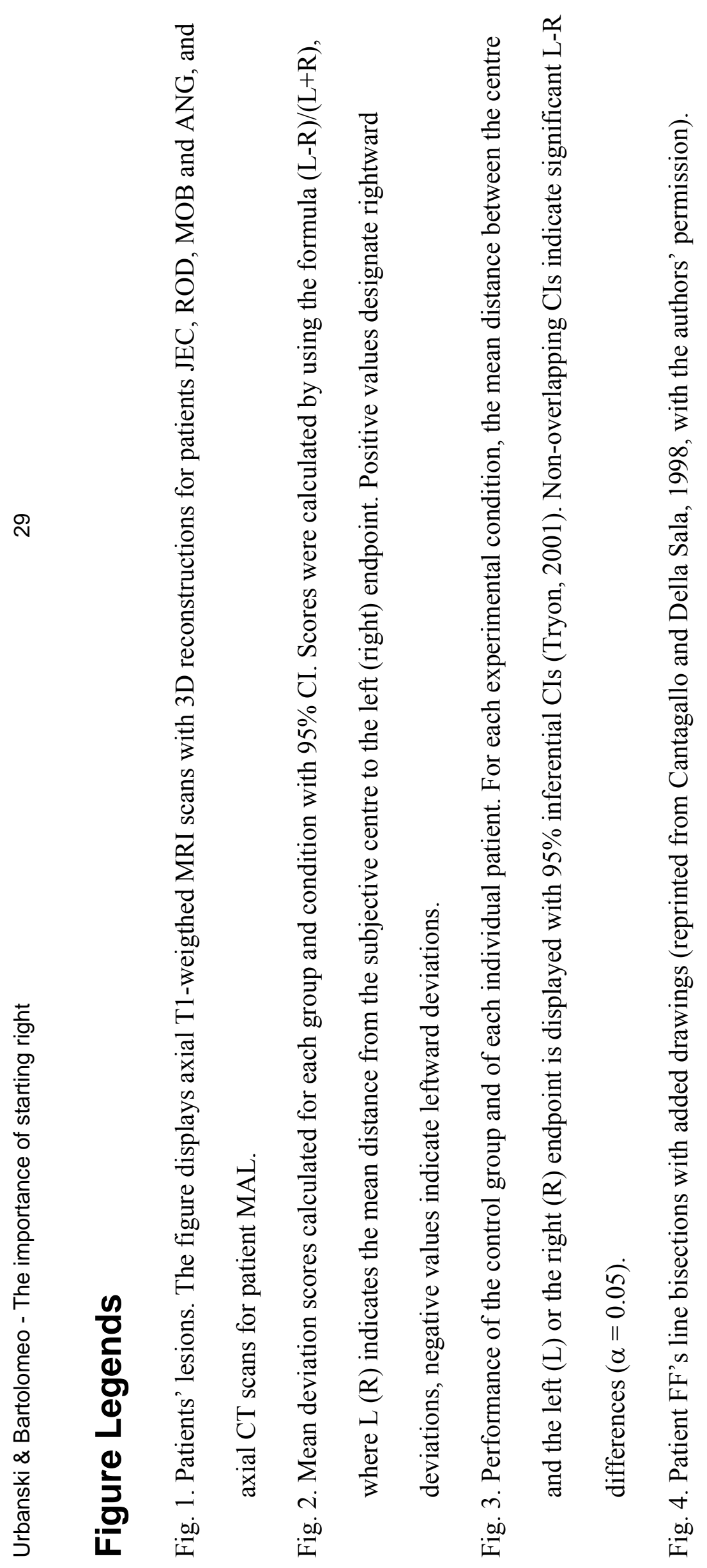


옴

픽

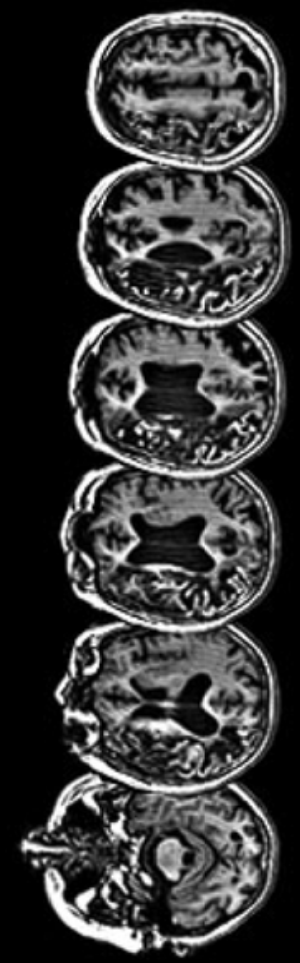




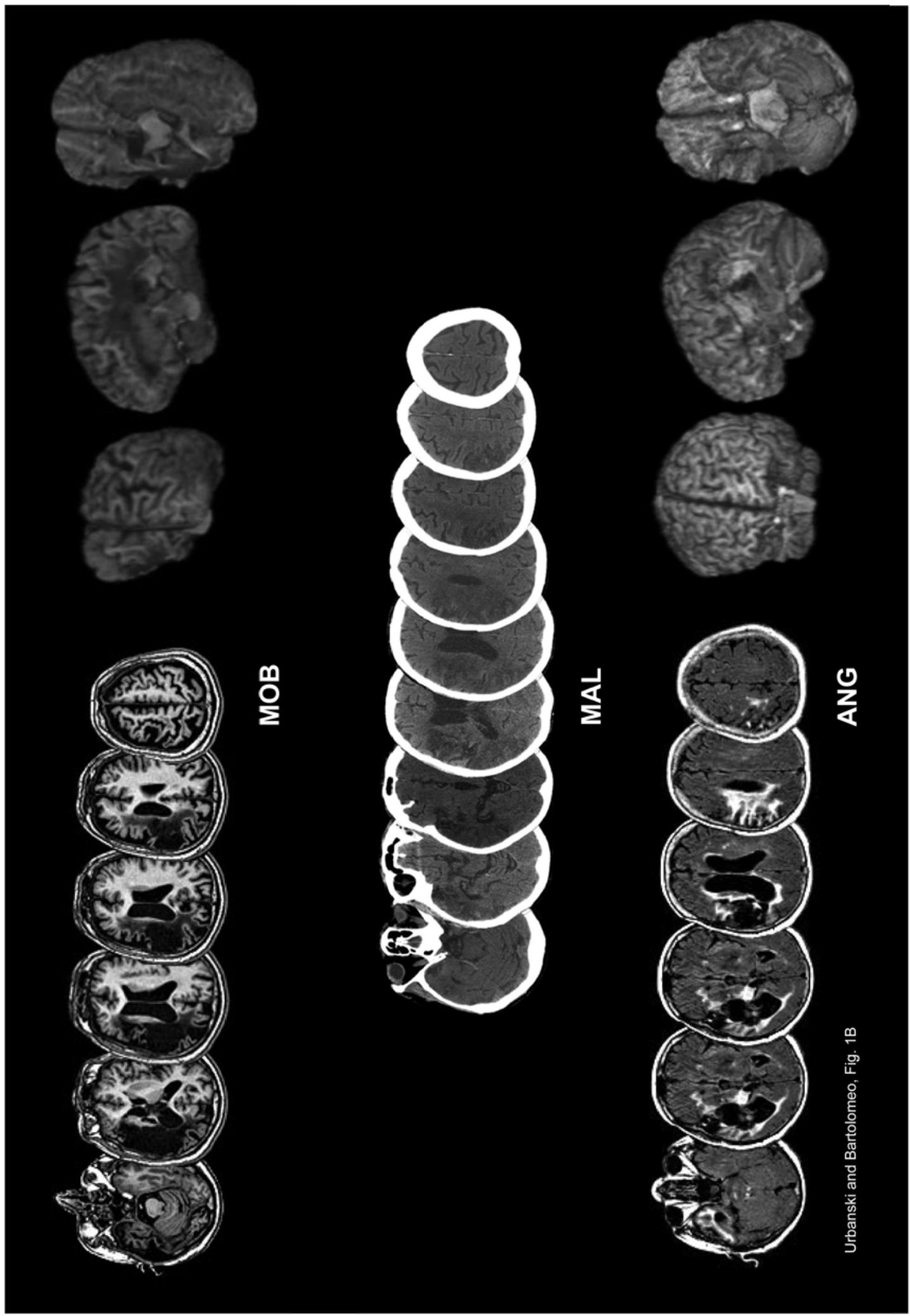




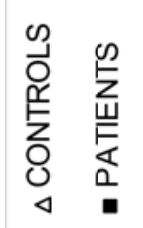

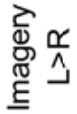

H
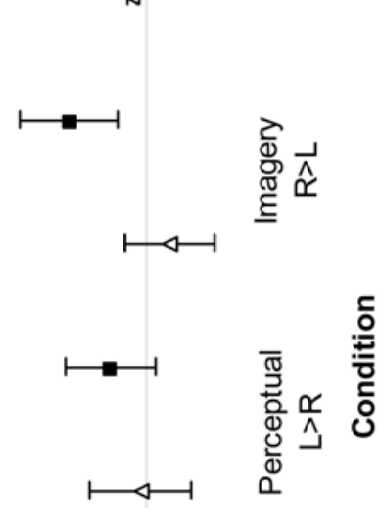

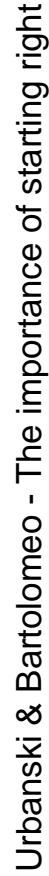

잉

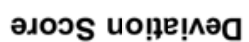




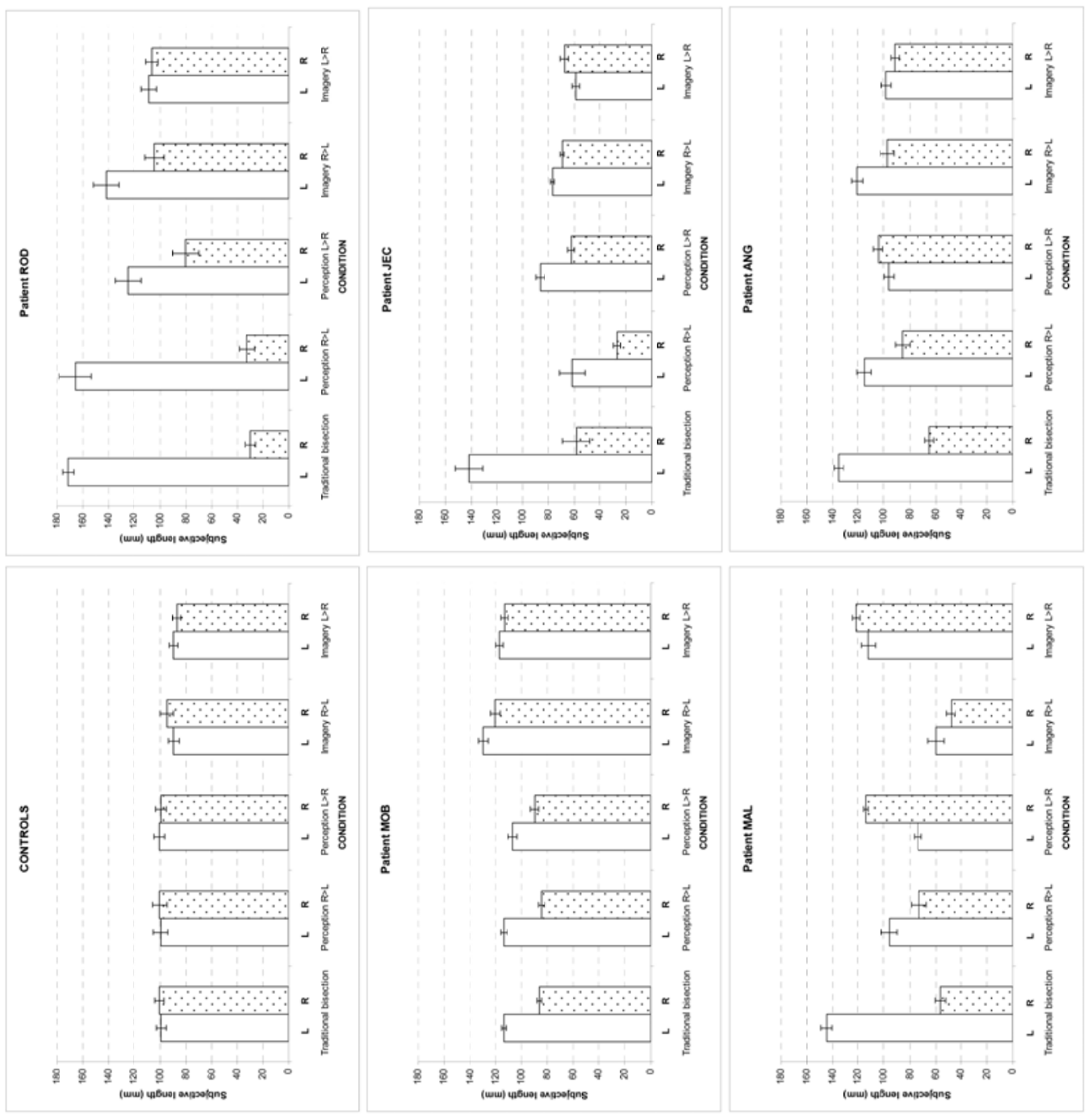


ले
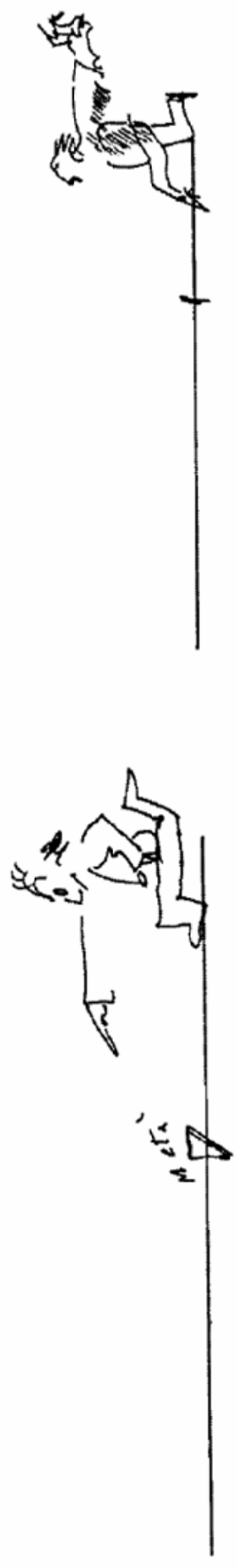

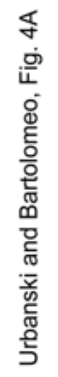




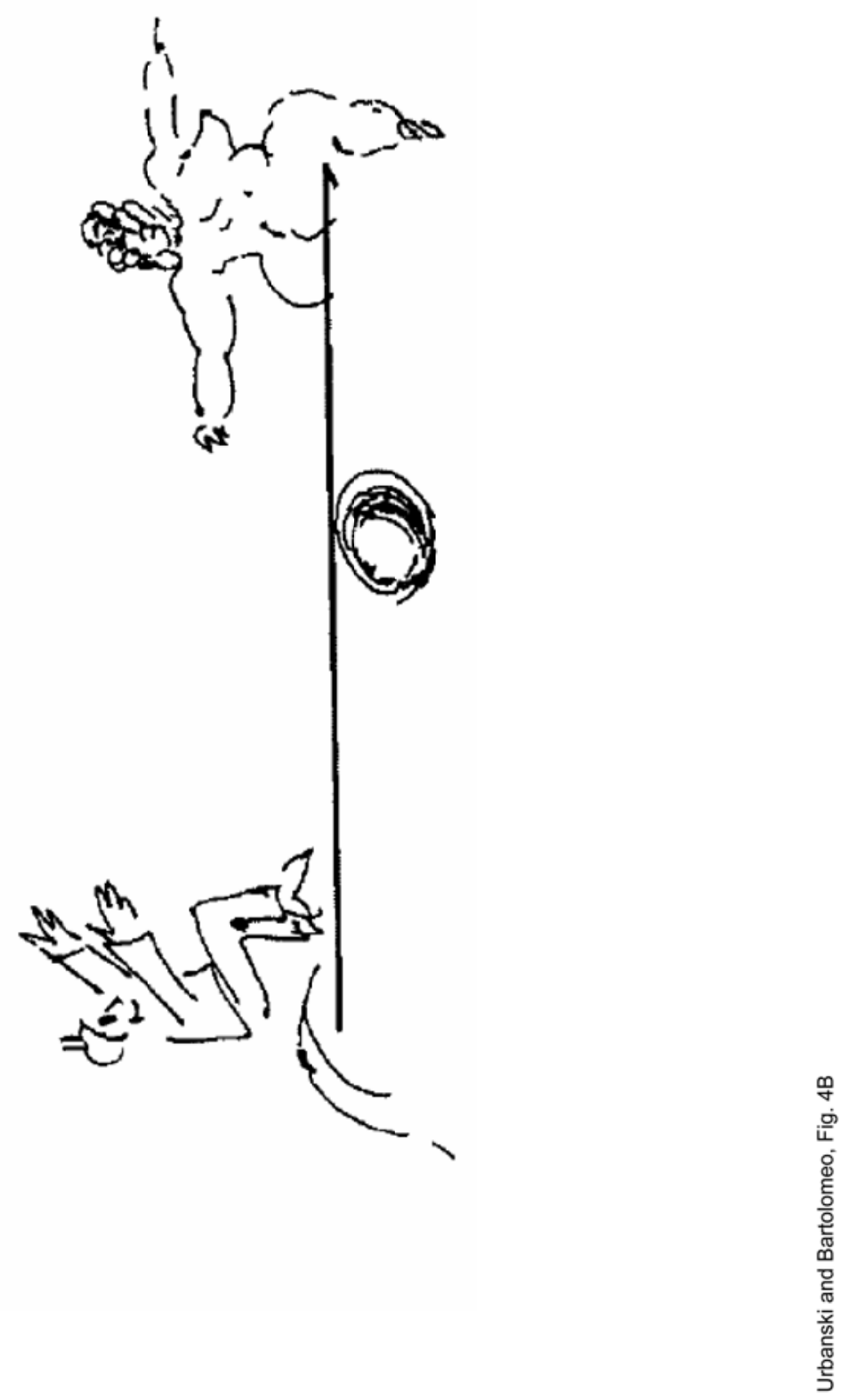

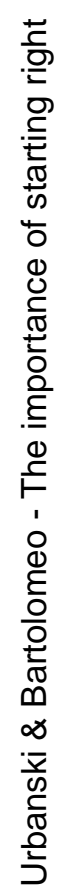

